\title{
Quiste broncogénico intramural esofágico
}

\author{
Intramural esophageal bronchogenic cyst \\ Lucas Panichelli*, Walter Salinas, Daniel Napolitano, Franco Signorini y Juan Muñoz \\ Servicio de Cirugía General, Hospital Privado Universitario de Córdoba, Córdoba, Argentina
}

\section{Resumen}

Antecedentes: Los quistes broncogénicos esofágicos son sacos cerrados originados de brotes anormales del tubo traqueobronquial primitivo. Estas lesiones suelen ser asintomáticas, por lo que pueden hallarse incidentalmente o pueden causar síntomas por compresión de estructuras adyacentes. Son lesiones muy poco comunes; se han publicado unos 23 casos en adultos. Caso clínico: Presentamos el caso de un varón de 44 años con un cuadro de tos de larga duración, sin otro síntoma. Para descartar las causas más comunes de sus síntomas se realizan estudios que objetivan la presencia de una lesión intramural de $40 \times 43 \mathrm{~mm}$. Se realiza videotoracoscopia para su resección y estudio, resultando ser un quiste broncogénico.

Palabras clave: Esófago. Leiomioma. Quiste broncogénico. Quiste esofágico.

\begin{abstract}
Background: Bronchogenic cysts are close sacks originated from abnormal development of the tracheobronchial tree. These lesions are usually asymptomatic or can cause symptoms, by compression of adjacent structures. This pathology is so unusual that there are only about 23 cases published in adults. Case report: We report a case of a 44 year-old man with persistent cough, without other symptom. The most common causes for his symptoms were ruled out, so imaging studies were made, showing at the medial portion of the esophagus, located intramural a $40 \times 43 \mathrm{~mm}$ ovoid lesion. Resected by video-assisted thoracoscopy for its study. With findings attributable to bronchogenic cyst.
\end{abstract}

Key words: Esophagus. Leiomyoma. Bronchogenic cyst. Esophageal cyst.

\section{Introducción}

Los quistes broncogénicos son sacos cerrados que se originan de brotes anormales del tubo traqueobronquial primitivo. Son raras lesiones congénitas que aparecen por fallas durante la formación del intestino anterior y son más comunes en el mediastino medio'.
Los estudios demográficos indican que estas lesiones tienen predominio en el sexo femenino (65.2\%). Los quistes puramente intramurales de esófago son hallazgos muy poco comunes, sobre todo en adultos ${ }^{2}$.

Estas lesiones son usualmente asintomáticas, por lo que pueden hallarse incidentalmente o pueden causar síntomas por compresión de estructuras adyacentes o por complicaciones.

\section{Correspondencia:}

*Lucas Panichelli

Naciones Unidas, 346

Parque Vélez Sarsfield

Fecha de recepción: 21-11-2019

C.P. 5000, Córdoba, Argentina

E-mail: lucaspanichelli@gmail.com
Cir Cir. 2020;88(S1):94-97

Contents available at PubMed www.cirugiaycirujanos.com (http://creativecommons.org/licenses/by-nc-nd/4.0/) 
El diagnóstico preoperatorio de estas lesiones es difícil de realizar, a pesar de la gran utilidad de los estudios de imagen para su caracterización, y se requieren métodos de anatomía patológica para confirmarlo'.

Reportamos un caso de quiste broncogénico intraesofágico escindido por videotoracoscopia.

\section{Caso clínico}

Varón de 44 años con antecedente de hipertensión arterial y tabaquismo, que se presenta a la consulta con tos crónica, no productiva, sin presentar disnea, disfagia, pérdida de peso ni otro síntoma acompañante. La exploración física no demostró alteraciones y los estudios de laboratorio estuvieron dentro de los valores normales. Luego de haber descartado causas más probables de sus síntomas, se decide realizar tomografía computada (TC) (Fig. 1) y resonancia magnética (RM), en las que se objetiva, en el tercio medio de la pared lateral derecha del esófago, en localización intramural, una imagen ovoidea, de bordes definidos, de aproximadamente $40 \times 43 \mathrm{~mm}$, con escaso refuerzo ante la inyección de contraste en la TC, de densidad de partes blandas, que sugiere como primera opción un leiomioma. Se realiza videoendoscopia digestiva alta, en la cual no se observa ninguna alteración de la mucosa ni de la anatomía normal del esófago.

Al sospecharse una lesión benigna, no se solicitan marcadores tumorales.

Se realiza un abordaje videotoracoscópico con el paciente en posición de decúbito lateral izquierdo, con inclinación de $45^{\circ}$, exponiendo el hemitórax derecho posterolateralmente, y se realizan tres incisiones de $15 \mathrm{~mm}$ : una a nivel del sexto espacio intercostal por debajo del vértice de la escápula, otra en el cuarto espacio intercostal por encima de la escápula, y la tercera en el noveno espacio intercostal en la misma línea del trocar previo. Se observó la tumoración entre los tercios distal y medio del esófago, y se proyectaba hacia la línea derecha del mismo. Se resecó realizando una apertura de los planos musculares, sin lesión de mucosa, presentando importantes adherencias a los planos adyacentes en la porción medial, por lo que se termina de resecar con un disparo de sutura mecánica de $45 \mathrm{~mm}$ púrpura. Se cierra por planos con Vicryl ${ }^{\circledR} 2-0$. Se envía a anatomía patológica una pieza de $33 \times 15 \mathrm{~mm}$ de características quísticas, con mucosidad espesa en su interior, la cual drena su contenido, y se coloca drenaje pleural y mediastinal. El

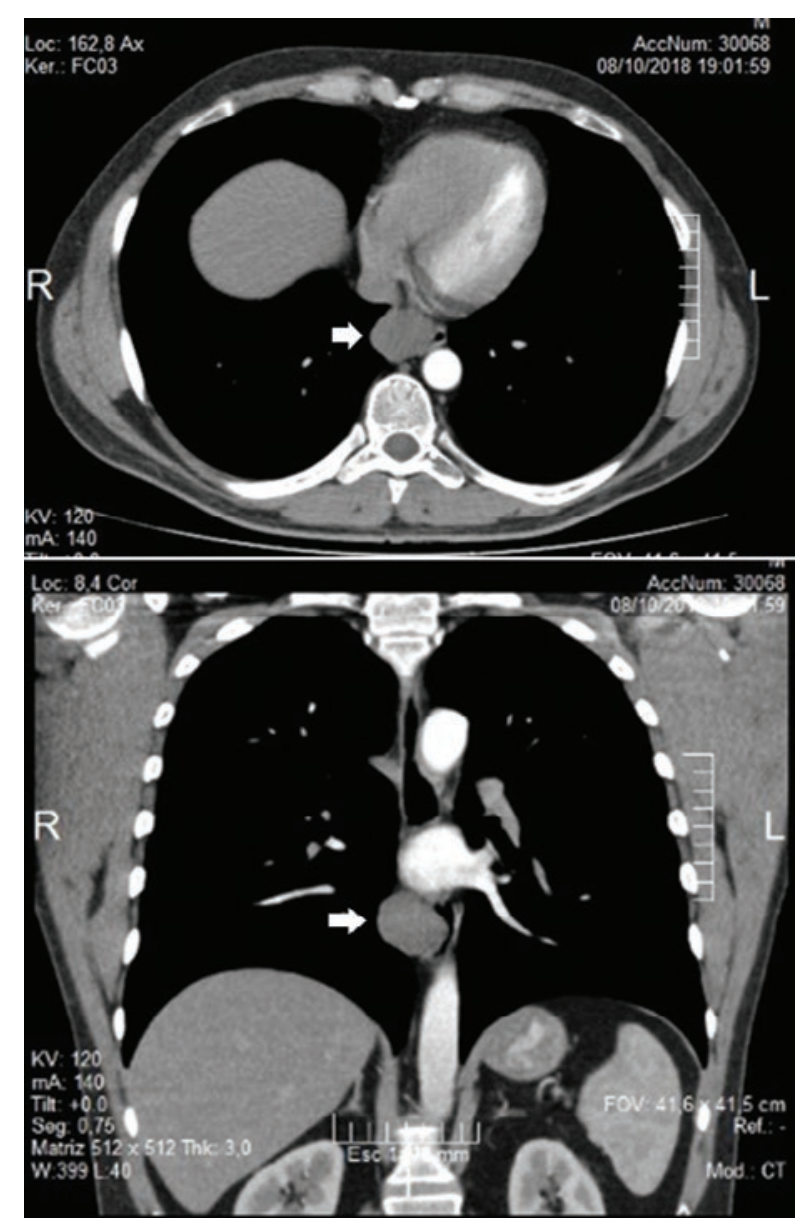

Figura 1. Cortes tomográficos de la lesión (flechas).

paciente cursa su posoperatorio inmediato en sala común. Presenta buena evolución y se realizan radiografías de control en los días siguientes. Se egresa al quinto día de posoperatorio y acude a control al mes de la cirugía sin haber presentado ninguna complicación.

Los cortes histológicos muestran la pared del quiste revestido en sectores por epitelio respiratorio que asienta sobre tejido fibroconectivo y muscular liso, observándose además focos de cartílago maduro (Fig. 2). Estos estos hallazgos son compatibles con quiste broncogénico.

\section{Discusión}

Los quistes broncogénicos son lesiones poco comunes, siendo el $10-15 \%$ de las masas primarias del mediastino, en relación con el árbol bronquial o el parénquima pulmonar, o paraesofágicos. Fueron descritos por primera vez en 1911 por Blackader, y Maier reportó en 1948 la primera resección quirúrgica ${ }^{3}$. 


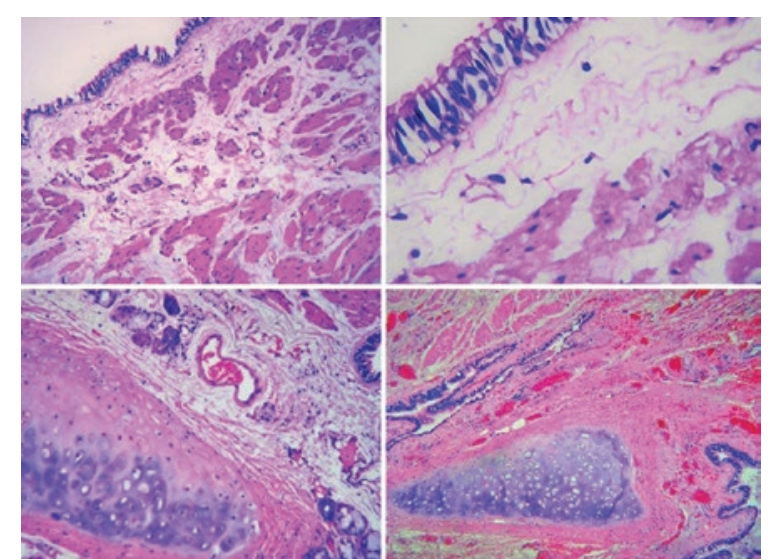

Figura 2. Cortes histológicos que muestran epitelio respiratorio. C y D: se observa cartílago maduro.

Los quistes broncogénicos resultan de un brote anormal del intestino anterior, evento que ocurre entre los días 26 y 40 de gestación ${ }^{4}$. Dependiendo de esto es la ubicación donde se les puede identificar, siendo el mediastino medio, cercano a la carina, la localización intratorácica más común (36-90\%) tinales usualmente están adyacentes al tercio distal de la tráquea o proximales al bronquio principal; se dividen en pericarinales (52\%), paratraqueales (19\%), paraesofágicos (14\%) y retrocardiacos $(9 \%)$. Los quistes puramente intramurales de esófago son lesiones muy poco comunes; solo hay publicados unos 23 casos en adultos desde $1981^{2}$.

Los pacientes suelen permanecer asintomáticos, pero en caso de presentar síntomas, estos son producidos por compresión de las estructuras circundantes o por las complicaciones relacionadas con el quiste ${ }^{6}$. Los síntomas más frecuentes son dolor torácico, tos, fiebre y disnea. En ocasiones pueden presentar complicaciones, tales como bronquitis, neumonía, pericarditis, sepsis, disfonía, disfagia, hemoptisis o malignización ${ }^{7}$. Por esto fue que en nuestro caso se sospechó una patología pulmonar como principal causa de los síntomas y se realizó una TC, en la que se evidenció la presencia de esta lesión esofágica, cambiando así nuestro enfoque diagnóstico.

En cuanto al diagnóstico, se pueden utilizar diferentes métodos de imagen. Los principales son la TC y la $\mathrm{RM}$, que resultan útiles para llevar a cabo el diagnóstico del quiste y descartar diagnósticos diferenciales, como duplicación esofágica, leiomioma, fibroma pleural, adenopatías o tumores malignos, entre otros ${ }^{1}$. Típicamente se observan como masas esféricas de densidad igual a la del agua o la del tejido blando ${ }^{3-8}$.
La RM es más sensible que la TC para diferenciar la localización y mostrar las características exactas. Los estudios reportan que la exactitud de la TC es del $69.2 \%$, mientras que la de la RM es del $100 \% 7$.

Las diferentes presentaciones de los quistes pueden llevar a incrementar la dificultad diagnóstica, puesto que si están infectados o tienen un alto contenido de proteínas su densidad aparenta ser mucho mayor y pueden confundirse con tejidos blandos 0 tumoraciones sólidas. Utilizando unidades Hounsfield (UH) se han descrito casos en los que el quiste presenta valores de $35 \mathrm{UH}$, característicos de tejidos sólidos (el agua presenta un valor de $0 \mathrm{UH}$ y los tejidos sólidos entre 20 y $70 \mathrm{UH})^{7}$. En nuestro caso, la alta densidad del contenido del quiste desde un primer momento dificultó el diagnóstico e hizo sospechar que la lesión en cuestión era un leiomioma de esófago.

También se puede realizar una videoendoscopia digestiva alta, cuyo principal interés está en los tumores de la mucosa y en la toma de biopsia. En cambio, en los tumores de la pared que no afectan a la mucosa se observa la deformidad provocada por la lesión submucosa ${ }^{8}$. Esto llevó a dudas en cuanto a si la lesión presentada por nuestro paciente era o no un leiomioma, ya que estos se ven por endoscopia como masas submucosas móviles, sobreelevadas, recubiertas por mucosa normal y, como ya se ha dicho, en la videoendoscopia digestiva alta realizada a nuestro paciente se observaba un esófago anatómicamente normal ${ }^{9}$.

Otros estudios también son útiles, como la ecoendoscopia, la cual es sensible para distinguir formaciones quísticas de masas sólidas, y facilita el diagnóstico de lesiones esofágicas. Permite también la toma de muestra por punción para determinar el contenido. Sin embargo, la gran variación en las formas de los quistes, la dificultad técnica del estudio y su poca disponibilidad en ciertos países hacen que no se recomiende realizarlo sistemáticamente, sino solo en casos de duda diagnóstica'.

En cuanto a los marcadores tumorales, deberían estudiarse ante la sospecha de malignidad de la masa; los principales son CA19.9 y CA 125. Su positividad es rara en esta patología ${ }^{7}$; solo hemos encontrado dos casos en los que los marcadores fueron positivos $^{1-7}$. En nuestro paciente, al sospechar que la lesión era benigna no se consideró necesario determinar los marcadores.

En cuanto al manejo, la escisión completa es recomendable, tanto por toracotomía como por 
videotoracoscopia, aunque el quiste sea asintomático, más que nada para evitar posibles complicaciones.

Según los datos obtenidos, la técnica más utilizada para la resección de estas lesiones es la toracotomía ${ }^{5}$. Hoy en día es recomendable la realización de procedimientos poco invasivos, como la videotoracoscopia, por sus ventajas con respecto a la toracotomía:

- Mejor visualización dentro de la cavidad torácica sin la necesidad de una gran incisión (sumado a la poca vascularización que suelen presentar estos quistes).

- Importante reducción en el dolor posoperatorio, puesto que las incisiones son mucho menores (aunque hay que aclarar que la cirugía sigue siendo un procedimiento doloroso que requiere buen control analgésico).

- Menos pérdida sanguínea.

- Alta hospitalaria más temprana, vuelta a la vida normal más precoz y rápida reinserción laboral, por lo que los costos se reducen.

\section{Conclusión}

Los quistes broncogénicos intramurales del esófago son lesiones extremadamente raras y poco estudiadas por su baja presentación. Son de difícil diagnóstico preoperatorio, presentando múltiples diagnósticos diferenciales con patologías mediastínicas y esofágicas, por lo que son de gran utilidad los métodos de diagnóstico por la imagen y el examen anatomopatológico.

Como tratamiento, la mejor opción es la escisión quirúrgica, para evitar cualquier complicación futura. Hoy en día, las técnicas videoasistidas deberían ser consideradas como primera opción por su ventajas en comparación con las técnicas convencionales, quedando esto supeditado a las capacidades de cada cirujano.

\section{Conflicto de intereses}

Los autores del trabajo niegan presentar conflictos de intereses para la realización del presente trabajo.

\section{Responsabilidades éticas}

Protección de personas y animales. Los autores declaran que para esta investigación no se han realizado experimentos en seres humanos ni en animales.

Confidencialidad de los datos. Los autores declaran que han seguido los protocolos de su centro de trabajo sobre la publicación de datos de pacientes.

Derecho a la privacidad y consentimiento informado. Los autores han obtenido el consentimiento informado de los pacientes y/o sujetos referidos en el artículo. Este documento obra en poder del autor de correspondencia.

\section{Bibliografía}

1. Cahoqun $H$, Rong $L$, Jun $Y$, Qin Z, Yang Z, Jun L, et al. A case report of esophageal bronchogenic and review of the literature with an emphasis on endoscopic ultrasonography appearance. Md-Journal. 2016;95: 1-6.

2. Altieri MS, Zheng R, Pryor AD, Heimann A, Ahn S, Telem DA. Esophageal bronchogenic cyst and review of the literature. Surg Endosc. 2015;29:3010-5.

3. Nolasco-de la Rosa AL, Nuñez-Trenado LA, Román-Guzmán E, Chávez-Villicaña CE. Neck bronchogenic cyst. Case report and review of the literature. Cir Cir. 2016;84:235-9.

4. Yesilkaya Y, Gulek B, Duymus M. Paraesophageal bronchogenic cyst mimicking oesophageal tumour. N Z Med J. 2012;126:79-81.

5. Turkyilmaz A, Eroglu A, Subasi M, Findik G. Intramural esophageal bronchogenic cysts: a review of the literature. Dis Esophagus. 2007;20: 461-5.

6. Gosal PKS, Mumme A, Harrison R, Williams N. Intramural oesophageal bronchogenic cyst: a rare pathological finding. ANZ J Surg. 2017;87): 1059-60.

7. Cheng Y, Chen D, Shi L, Yang W, Sang Y, Duan S, et al. Surgical treatment of an esophageal bronchogenic cyst with massive upper digestive tract hematoma without esophagectomy: a case report and the review of the literature. Ther Clin Risk Manag. 2018;14:699-707.

8. Galindo F, Marzano C, De La Torre A. Tumores benignos del esófago. Enciclopedia Médica Americana. 2006;1(180):1-8.

9. Loviscek L, Hyoun Yun J, Soon Park Y, Chian A, Grillo C, Cenoz M. Leiomioma de esófago. Cir Esp. 2009;85(3):147-51. 